\title{
Optical pathlength enhancement in ultrathin silicon solar cell using decorated silver nanoparticles on aluminium grating
}

\author{
S. Saravanan \\ Advanced Research Laboratory for Nanomaterials \& Devices, Department of Nanotechnology Swarnandhra College \\ of Engineering \& Technology, Seetharampuram, Narsapur 534 280, West Godavari, Andhra Pradesh, India \\ shasa86@gmail.com
}

PACS 73.50.h, 84.60.Jt

DOI 10.17586/2220-8054-2020-11-1-86-91

In this work, ultrathin silicon solar cell design employed with the aid of periodic silver (Ag) nanoparticles substituted on an aluminium (Al) grating to improve the optical performance by using rigorous coupled-wave analysis (RCWA) method. The enhanced light absorption was observed in the silicon absorber region, due to the photonic and plasmonic modes between the metal and dielectric surface. With the optimal structure, maximum short-circuit current densities were observed at transverse magnetic $\left(\sim 36.13 \mathrm{~mA} / \mathrm{cm}^{2}\right)$ and electric $\left(31.59 \mathrm{~mA} / \mathrm{cm}^{2}\right) \mathrm{modes}$. Further, we have demonstrated the effectiveness of the different ultrathin silicon solar cells with plasmonic structures and compared.

Keywords: Ultrathin, plasmonic, absorption, grating, nanoparticle, current density.

Received: 28 November 2019

Revised: 25 December 2019

\section{Introduction}

Nowadays, photovoltaic (PV) industries have been drawn great interest in silicon-based materials due to their natural abundance, low cost, long-term stability with well-equipped technology. These advanced technologies have the capability to yield higher conversion cell efficiency with better stability and researchers are continuously working in that area. Particularly, the nanostructure or thin-film material is appropriate and holding promises to enhance optical performance in PV devices [1]. However, the light trapping mechanism plays a crucial role in the solar cells and improving optical properties due to the various nanostructure such as a nanoparticle, nanorod, distributed Bragg reflector (DBR), nanograting (metal/dielectric) and different refractive indexed nanostructures [2-6]. Among these, noble metal nanoparticles and nanogratings are evidenced by the strong absorption enhancement in the visible and IR spectral region. It can change the optical properties easily by inducing the charge carrier generation. Past few decades, researchers are concentrating on the noble metal nanoparticles ( $\mathrm{Au}, \mathrm{Ag}, \mathrm{Al}, \mathrm{Cu}$ ) in solar cells and excessively studied their performance [7-9]. Sidharthan and Murugesan explored the improved light absorption in the thin-film solar cell using an aluminium (Al) grating and silver (Ag) nanoparticles. The significant improvement noticed in the light absorption due to the combination of metal nanostructures. They have reported $\sim 5.4 \%$ enhanced absorption noticed when compared to the planar metal structure [10]. Zhang and Gu numerically explained the plasmonic light trapping effect in the ultrathin silicon solar cell with metal back reflectors using the finite difference time domain (FDTD) method. The maximum light absorption achieved at longer wavelengths due to the surface excitation and plasmon-coupled guided-mode resonance. The reference cell yields a current density of $\sim 3.55 \mathrm{~mA} / \mathrm{cm}^{2}$ and the silver integrated thin-film silicon solar cells achieved $\sim 6.8 \mathrm{~mA} / \mathrm{cm}^{2}$ within $100 \mathrm{~nm}$ thin silicon absorber layers. This investigation generated a significant effect on the plasmonic light-trapping structure-based solar cells [11]. Sathyamoorthy et al. studied the localized surface plasmon on a gold nanoparticles and found optimum thickness. The enhanced plasmonic effects were observed due to the size and shape of the dielectric medium around the gold nanoparticles. They demonstrated the gold nanoparticles were exhibited maximum plasmonic enhancement at $532 \mathrm{~nm}$ wavelength range with $60 \mathrm{~nm}$ size of the nanoparticles. Further, the Rayleigh scattering study was carried out with their experimental results [12]. Kakavelakis et al. investigated the efficiency and stability of perovskite solar cells by the addition of noble metal nanoparticles. They demonstrated the Ag and Au nanoparticles were providing their maximum power conversion efficiency. Similarly, the Al nanoparticles based solar cell device shown remarkably improved stability as compared others and achieved efficiency up to $13.5 \%$ [13]. Enrichi et al. explored the various plasmonic enhanced solar cells with possible strategies with the results. They reported that the performance of solar cell was determined by the limiting factors, for example, reducing size (layers/particles), acceptance angle of active region, active materials, adopting light trapping schemes and exploiting spectral modification. Furthermore, the plasmonic nanostructure has been boosting by scattering and concentrating the electromagnetic field towards the active region in the device. The different plasmonic approaches were discussed and compared, also provided a few key conclusions 
to understand the goal and future perspective of the field [14]. Morawiec et al. investigated the optical properties of localized surface plasmon resonance using self-assembled Ag nanoparticles (NPs) for photovoltaic applications. They fabricated Ag NPs on various substrates by the solid-state dewetting method and demonstrated the structural and optical properties of prepared nanostructures. The obtained spherical and uniform NPs were integrated as plasmonic backside reflector. The parasitic absorption in thin absorber deposited on the backside reflector measured using optoelectronic spectroscopic techniques [15].

In the proposed work, the ultrathin silicon solar cell performance was studied by using plasmonic backside reflectors. In section second, explained the designing approach of the complete solar cell. The current density, absorption spectrum, transverse electric (TE) and magnetic (TM) field analysis of optimal solar cell results were studied in section third. Finally, section fourth concludes the paper.

\section{Designing approach}

Figure 1 shows the illustration of ultrathin silicon solar cell and investigated using the RCWA method. In this simulation, the work was performed using the RCWA method, which is also known as a simple and fast technique. Initially, $50 \mathrm{~nm}$ thick layer of indium-tin-oxide (ITO) acting as an antireflection coating (ARC), silicon absorber with 50 and $100 \mathrm{~nm}$ thick aluminium (Al) layer acting as a substrate. On the metal substrate, Al grating added with $50 \mathrm{~nm}$ height $(\mathrm{Gh})$ and $100 \mathrm{~nm}$ width $(\mathrm{Gw})$ to scatter light at a larger angle. Because the metal nanogratings offer an opportunity for improving the electromagnetic (EM) fields near the surfaces by surface plasmon polariton [16].

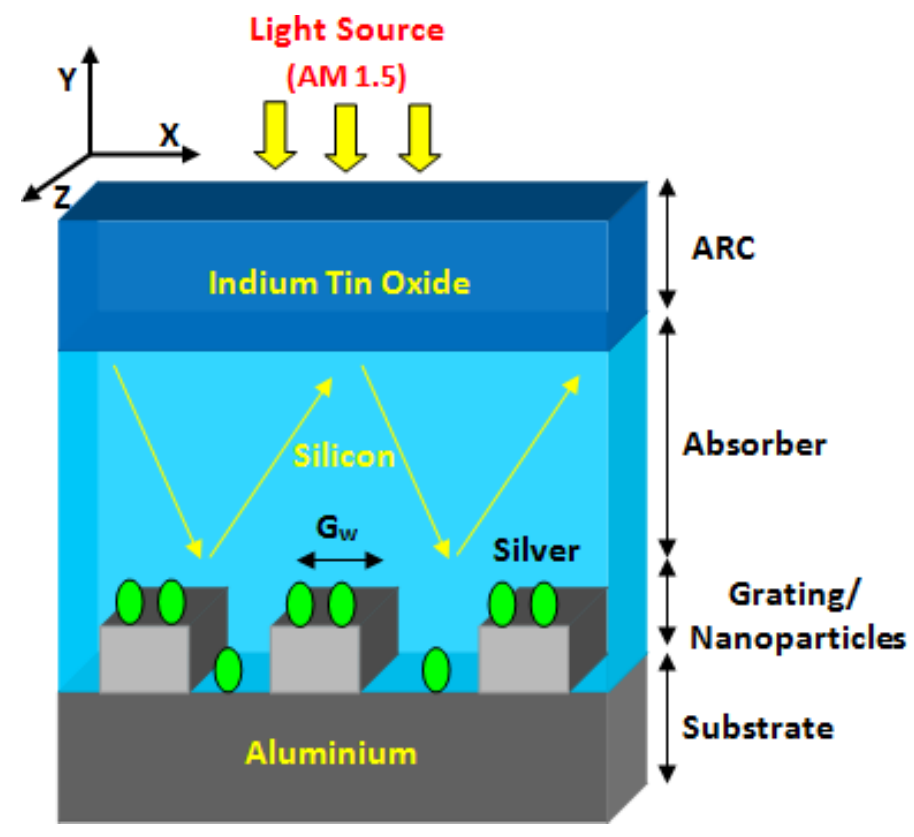

FIG. 1. The schematic diagram of ultrathin silicon solar cell

Furthermore, the $20 \mathrm{~nm}$ diameters silver $(\mathrm{Ag})$ nanoparticles periodically substituted on the $\mathrm{Al}$ gratings to enhance light trapping mechanism. Shilpa et al. reported the nano-sized (or nanoparticles) structure enhanced the conversion efficiency of silicon thin-film solar cells. They demonstrated the highest absorption achieved by optimal geometrical parameters of Ag nanoparticles than the Au nanoparticles [17]. Probably, the silver nanoparticle is the suitable material to scatter the incident light into the absorber region as compared to other noble metals like gold (Au) and aluminum (Al). The light absorption was expected to be improved due to Al grating and Ag nanoparticles within the solar cell. Recently, various methods are available in the market to study the enhancing optical absorption and the conversion efficiency of PV devices [18]. Our simulation structure was terminated by two boundary conditions, first periodic boundary condition (PBC) is employed (parallel) in $x$ - and $y$-axis, another perfect matched layer (PML) condition at $z$-axis and it has perpendicular to the light propagation direction. Here, the smallest spatial grid size $(10 \mathrm{~nm})$, harmonics (5) used throughout the simulation to maintain the accuracy of the RCWA calculations. Further, the designed solar cell structure extended and comparative studies are carried out using four different ultrathin film cells. 


\section{Results and discussion}

To compare the performance of various solar cell structures, hereafter named as solar cell A: ARC only, B: ARC+ Substrate, C: ARC+ NPs+ Substrate, D: ARC+ GRA+ Substrate and E: ARC+NPs+GRA+Substrate. Figure 2 depicted the short-circuit current density $\left(J_{s c}\right)$ of the different ultrathin film silicon solar cell.

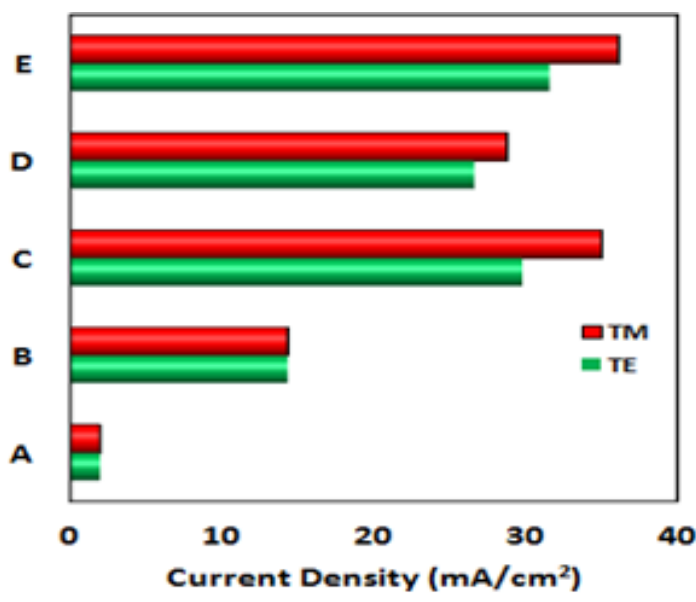

FIG. 2. The types of solar cell vs. short-circuit current density $\left(\mathrm{mA} / \mathrm{cm}^{2}\right)$

The short-circuit current density $\left(J_{s c}\right)$ associated with the number of light photons absorbed by the silicon absorber which could be generating the electron-hole pair combination and the charge carriers contributing the enhancement of current density [19]. Here, the improvement of different solar cell performance noticed with respect to simulated structure. First, solar cell 'A' shows less current density as compared to the other designed cell structure. From the result, cell ' $E$ ' reveals the highest current density in both modes such as transverse electric $\left(\sim 31.59 \mathrm{~mA} / \mathrm{cm}^{2}\right)$ and transverse magnetic $\left(36.13 \mathrm{~mA} / \mathrm{cm}^{2}\right)$ fields due to the combination of $\mathrm{Ag}$ NPs and $\mathrm{Al}$ grating. The incorporation of metal nanostructures, the significant optical performance was noticed by inducing charge carrier generation and acts as a light scattering source [20-23].

Figure 3(a,b) shows the absorption spectrum plotted in both polarizations (TE \& TM) conditions against the incident wavelength for five different ultrathin film solar cells. In TE case, Fig. 3(a) represents increased light absorption spectrum in the ascending order like cell A, B, C, D and E. The solar cell 'A' composed of anti-reflection coating (ARC) with absorber shows better absorption from 370 to $400 \mathrm{~nm}$. Solar cell 'B' shows one sharp peak at $390 \mathrm{~nm}$ and enhanced broader curve noticed from 670 to $1110 \mathrm{~nm}$ due to planar Al substrate. Solar cell ' $C$ ' shows shifted the highest peak from 430 to $520 \mathrm{~nm}$ and the broader curve obtained significantly from 590 to $900 \mathrm{~nm}$ due to added Ag nanoparticles on the planar Al substrate. Similarly, the Ag nanoparticle replaced with Al grating and observed sharp or highest peak shifted at $520 \mathrm{~nm}$ but failed to prove a broader curve in longer wavelength region as shown in cell 'D'. Finally, cell ' $E$ ' depicts the sharp peak between the cells ' $C$ ' and ' $D$ ' because of the combined effect of metallic (grating \& nanoparticles) nanostructure.

Figure 3(b) depicts various solar cell absorption spectrum under TM polarization. In cell 'A' and 'B' absorption curve not enhanced considerably. However, metal nanoparticle (Cell C) and grating (cell D) based designed structure reveals sharp and broader peaks in visible and infrared wavelength due to plasmonic effects such as LSP and guidedmode resonance (GMR). Solar cell 'E' remarkably enhanced the collection of the photon and observed broader curve in the visible range due to metal grating and nanoparticle, but infrared region reduced the plasmonic effect as compared previous (C and D) two designed solar cell where there is a need for more optimization.

Overall, both polarization causes a significant decrease in the absorption into three modes such as 760, 930 and $1120 \mathrm{~nm}$. Stuart and Hall have demonstrated a smaller number of modes available in absorption spectra because of that enhanced absorption reduced significantly due to some circumstances [1]. However, the required light absorption to be enhanced due to suitable light trapping mechanism within ultrathin materials and it can improve beyond the $4 n^{2}$ limits [12].

Figure 4(a-c) shows the electric field distributions in the designed ultrathin silicon solar cells using the RCWA method. The field has a normal incident angle $\left(0^{\circ}\right)$ and light is parallel to the grating and nanoparticles. Here, the optical concept such as diffraction, scattering, reflection and interference of the light provoked the intensity modulation. The Fabry-Perot resonance and strong guided modes observed at the different incident wavelength $(420,510 \&$ $1160 \mathrm{~nm})$ as shown in Fig. 4(a-c). 


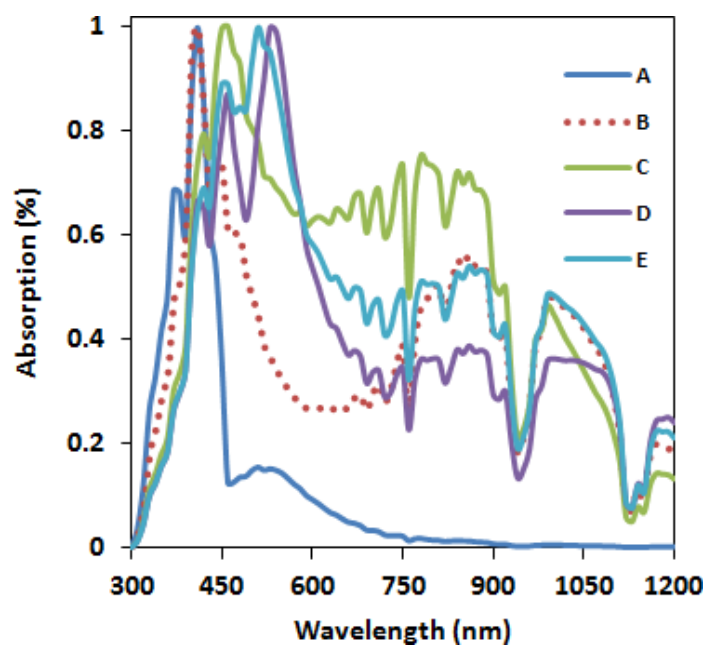

(a)

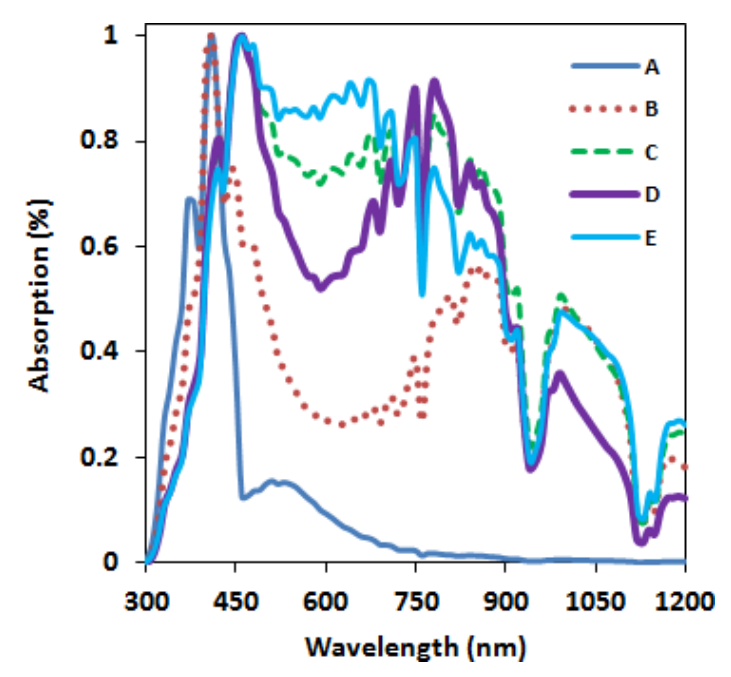

(b)

FIG. 3. Light absorption spectrum for TE (a), TM (b) polarization at different cell and comparison with standard solar spectrum $\left(\mathrm{Wm}^{-2} \mathrm{~nm}^{-1}\right)$ on the earths surface (c)

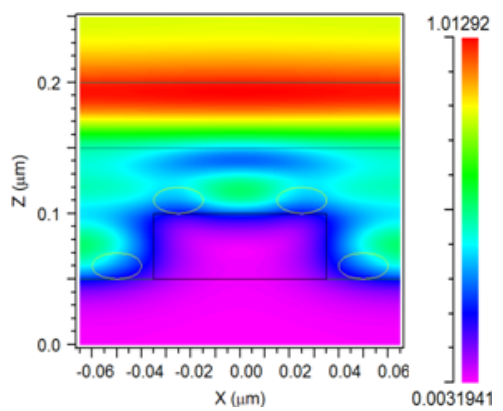

(a)

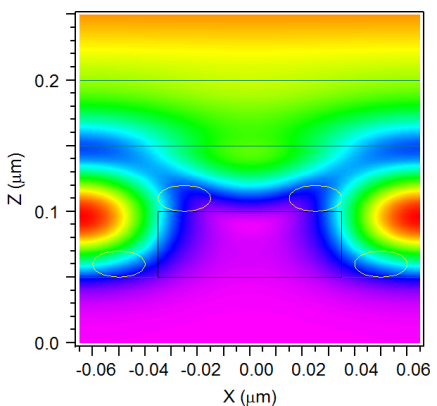

(b)
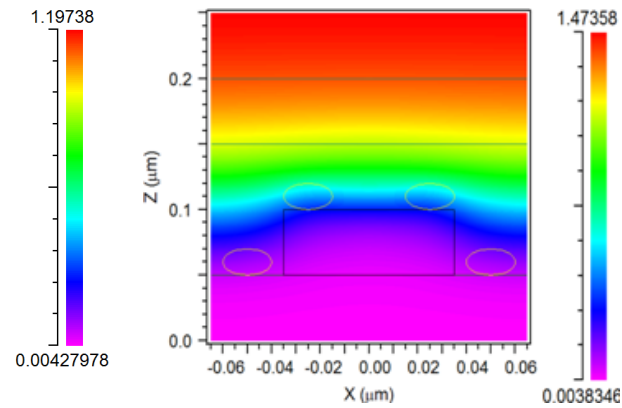

(c)

FIG. 4. Electric field intensity distributions with the guided modes at the wavelength (a) $420 \mathrm{~nm}$, (b) $510 \mathrm{~nm}$ and (c) $1160 \mathrm{~nm}$ with the normal incident

Figure 5 depicts the light interference between the ultrathin silicon solar cells and compared various incident center wavelengths. Here, the metal forces vanishing of the electric field within the tiny distance. For a conductor, the electric field should have a node (grating) with that the coherent (superposition) monochromatic light described as standing wave above the absorber [24]. Fig. 5(a-e) shows the magnetic field distribution of difference shorter and longer wavelength. Fig. 5(a) shows the guided modes in the absorber region and localized surface plasmon on the Ag nanoparticles which generated the maximum number of charge carrier at $420 \mathrm{~nm}$ incident wavelength. The visible wavelengths are 460 and $670 \mathrm{~nm}$ shows the strongly localized surface plasmon (LSP) appeared on the Al grating and Ag nanoparticles as revealed in Fig. 5(b,c). Remarkably, infrared wavelength (990 and $1120 \mathrm{~nm})$ indicates the strong LSP observed in Fig. 5(d) due to metallic nanostructures. Overall, the strong plasmonic effect shows through bright (red color) regions corresponding to the field intensity. The silver nanoparticles were supporting and boosting the collection of charge carrier absorption within the solar cells [9].

\section{Conclusion}

In conclusion, we have investigated the light trapping ability of Ag nanoparticle functionalized $\mathrm{Al}$ grating as back reflector in ultrathin silicon solar cell using RCWA method. The results shown enhanced optical performance in the visible and infrared region by accompanying the standing wave with in the absorber region. Finally, the optimized ultrathin silicon solar cell yielded the highest current density $\sim 31.59 \mathrm{~mA} / \mathrm{cm}^{2}$ (TE) and $\sim 36.13 \mathrm{~mA} / \mathrm{cm}^{2}$ (TM) within $50 \mathrm{~nm}$ silicon absorber region. Further, this work addressing the improved plasmonic effect in ultrathin film solar cells by using metal nanostructures with perfect forward scattering while getting rid of backward scattering. 


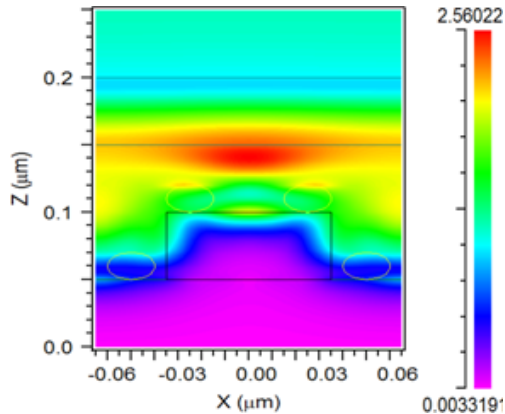

(a)

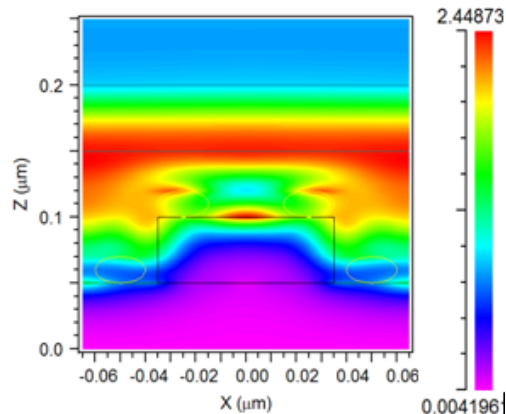

(b)

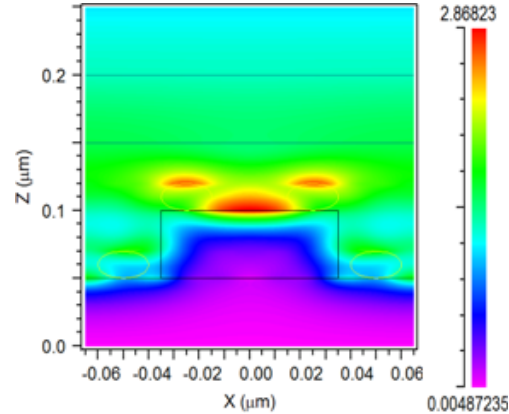

(c)

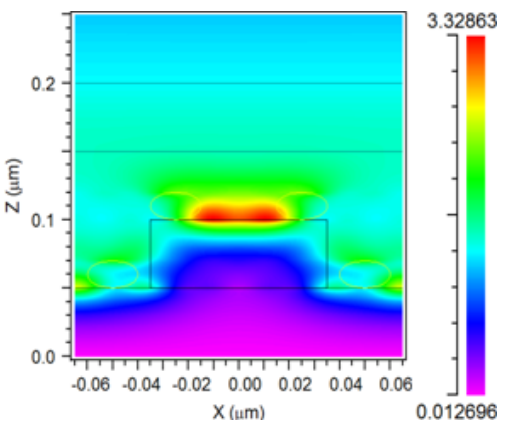

(d)

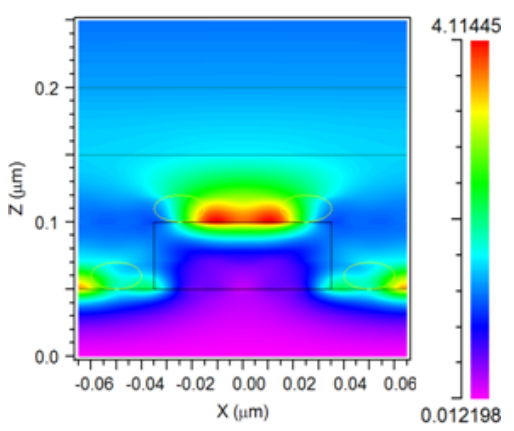

(e)

FIG. 5. The magnetic field intensity profiles at (a) $420 \mathrm{~nm}$, (b) $460 \mathrm{~nm}$, (c) $670 \mathrm{~nm}$, (d) $990 \mathrm{~nm}$ and (e) $1120 \mathrm{~nm}$

\section{References}

[1] Stuart H.R., Hall D.G. Thermodynamic Limit to Light Trapping in Thin Planar Structures. Journal of the optical society of America A, 1997, 14, P. 3001-3008

[2] Saravanan S., Dubey R.S. Optical absorption enhancement in $40 \mathrm{~nm}$ ultrathin film silicon solar cells assisted by photonic and plasmonic modes. Optics Communications, 2016, 377, P. 65-69.

[3] Enes Battal, Taha Alper Yogurt, Levent Erdal Aygun, Okyay Ali K. Triangular metallic gratings for large absorption enhancement in thin film Si solar cells. Optics express, 2012, 20, P. 9458-9464.

[4] Pillai S., Catchpole K.R., Trupke T., Green M.A. Surface Plasmon Enhanced Silicon Solar Cells. Journal of Applied Physics, 2007, 101, 093105-1-8.

[5] Saravanan S., Dubey R.S., et al. Design and optimization of ultrathin crystalline silicon solar cells using an efficient back reflector. AIP Advances, 2015, 5, 057160-1-9.

[6] Mariani G., Wong P.S., et al. Patterend radial GaAs nanopillar solar cells. Nano Letters, 2011, 11, P. $2490-2494$.

[7] Zhang Y., Stokes N., et al. Toward ultra-thin plasmonic silicon wafer solar cells with minimized efficiency loss. Scientific Reports, 2014, 4, P. 1-6.

[8] Sreekanth K.V., Sidharthan R. Murukeshan V.M. Gap modes assisted enhanced broadband light absorption in plasmonic thin film solar cell. Journal of Applied Physics, 2011, 110, 033107-1-5.

[9] Zhang Y., Cai B., Jia B. Ultraviolet plasmonic aluminum nanoparticles for highly efficient light incoupling on silicon solar cells. Nanomaterials, 2016, 6, P. 1-10.

[10] Sidharthan R., Murukeshan V.M. Improved light absorption in thin film solar cell using combination of gap modes and grating back reflector. Thin Solid Films, 2013, 548, P. 581-584.

[11] Zhang Y., Gu M. Plasmonic light trapping for wavelength-scale silicon solar absorbers. Frontiers of Optoelectronics, 2016,9 , P. $277-282$.

[12] Sathiyamoorthy K., Sreekanth K.V., et al. Surface plasmon enhancement in gold nanoparticles in the presence of an optical gain medium: an analysis. Journal of Physics D: Applied Physics, 2014, 44, 425102-1-9.

[13] Kakavelakis G., Alexaki K., Stratakis E., Kymakis E. Efficiency and stability enhancement of inverted perovskite solar cells via the addition of metal nanoparticles in the hole transport layer. RSC Advances, 2017, 7, P. 12998-13002.

[14] Enrichi F., Quandt A., Righini G.C. Plasmonic enhanced solar cells: summary of possible strategies and recent results. Renewable and Sustainable Energy Reviews, 2018, 82 (3), P. 2433-2439.

[15] Morawiec S., Mendes M.J., Priolo F., Crupi I. Plasmonic nanostructure for light trapping in thin-film solar cells. Materials Science in Semiconductor Processing, 2019, 92, P. 10-18.

[16] Alan X., Wang, Xianming K. Review of Recent Progress of Plasmonic Materials and Nano-Structures for Surface Enhanced Raman Scattering Materials. Materials, 2015, 8, P. 3024-3052.

[17] Shilpa G.D., Subramanyam T.K., Sreelakshmi K., Uttarakumari. Study and optimization of metal nanoparticles for the enhanced efficiency thin film solar cells. Materials Science Engineering, 2016, 149, 012074-1-6. 
[18] Kim R.S., Zhu J., et al. E-Beam deposited Ag-nanoparticles plasmonic organic solar cell and its absorption enhancement analysis using FDTDbased cylindrical nanoparticle optical model. Optics Express, 2012, 20, P. 12649-12657.

[19] Pudasaini P.R., Arturo A.A. Nanostructured thin film silicon solar cells efficiency improvement using gold nanoparticles. Physica Status Solidi $A, 2012,209$, P. 1475-1480.

[20] Lim S.P., Pandikumar A., et al. Promotional effect of silver nanoparticles on the performance of $\mathrm{N}^{-d o p e d ~} \mathrm{TiO}_{2}$ photoanode-based dyesensitized solar cells. RSC Advances, 2014, 4, P. 48236-48244.

[21] Lim S.P., Pandikumar A., Huang N.M., Lim H.N. Enhanced photovoltaic performance of silver titania plasmonic photoanode in dye-sensitized solar cells. RSC Advances, 2014, 4, P. 38111-38118.

[22] Yu B., Leung K.M., et al. Synthesis of Ag-TiO 2 Composite Nano Thin Film for Antimicrobial Application. Nanotechnology, 2011, 22, 115603-1-9.

[23] Shah S., Noor I.M., et al. Plasmonic effects of quantum size metal nanoparticles on dye-densitized solar cell. Optical Materials Express, 2017, 7 P. 2069-2083.

[24] Shore B.W., Feit M.D., et al. Electric field enhancement in metallic and multilayer dielectric gratings. SPIE, 1997, 2633, P. 709-714. 